\title{
Rectal Colonization with Extended Spectrum $\beta$-Lactamase producing Enterobacteriacieae in Surgical Patients in a Tertiary Hospital in Calabar, Nigeria.
}

\author{
Godwin Ibitham Ogban, ${ }^{1,2}$ Ernest Afu Ochang, ${ }^{1,2}$ Ubleni Ettah Emanghe, ${ }^{1}$ \\ Usang Edet Usang, ${ }^{3}$ Ubong Bassey Akpan, ${ }^{4}$ Thomas U. Agan ${ }^{4}$ \\ 1. Department of Medical Microbiology and Parasitology, University of Calabar Teaching Hospital, Calabar, \\ CRS, Nigeria. \\ 2. Save Lives Initiative (NGO) \\ 3. Department of Surgery, University of Calabar Teaching Hospital, Calabar, CRS, Nigeria. \\ 4. Department of Obstetrics and Gynaecology, University of Calabar Teaching Hospital, Calabar, CRS, Nigeria
}

\begin{abstract}
Aim: Extended spectrum $\beta$-lactamases are transferable plasmid mediated resistance mechanisms found mainly among Enterobacteriaceae which confer resistance to numerous $\beta$-lactam antibiotics. The aim of the study was to determine the rate of rectal colonization of surgical patients with Escherichia coli carrying extended spectrum $\beta$-lactamases. Subjects and methods: We collected and cultured rectal swabs from 192 surgical patients in the University of Calabar Teaching Hospital, Calabar, Nigeria. Escherichia coli isolates were identified and antibiotics susceptibility performed using Modified Kirby-Bauer disc diffusion technique. Isolates showing zones of inhibition to third generation cephalosporins below Clinical and Laboratory Standard Institute recommended break-points were screened for ESBL-production by the double-disk synergy method. Results: A total of 67.7\%(130) of the patients received prophylactic antibiotics which was continued empirically for $\geq 5$ days after surgery. Ceftriaxone was the most empirically prescribed antibiotic used with metronidazole in $63.1 \%(82 / 130)$ and alone in 7.7\%(10/130) of patients. Ceftriaxone resistance was observed in 39.6\%(76/192) of the rectal E. coli isolates with 3.1\%(6/192) showing intermediate susceptibility. Ceftazidime resistance was observed in 40.6\%(78/192) with 4.2\%(8/192) showing intermediate susceptibility. ESBL mediated resistance was observed in 27.1\%(52/192) of isolates. ESBL positive isolates were more likely to be resistant to Gentamicin, Ciprofloxacin and Co-trimoxazole than negative isolates $(P<0.001)$. All isolates were susceptible to Meropenem. Younger patients were more colonized with a preponderance in patients less than 9 years of age (Pearson's correlation $=-0.196 ; P=0.006)$. Conclusion: Infection may occur in colonized surgical patients leading to increased morbidity. Pre-surgical screening to identify colonized patients will be beneficial in the early selection of appropriate therapy and management of outbreaks while functional hospital antibiotic stewardship programmes and strict enforcement of national antibiotic regulations will curtail their spread.
\end{abstract}

Key words: ESBL, Escherichia coli, Hospital Acquired Infections, Infection Control, Surgical Sites Infections.

\section{Introduction}

Enterobacteriaceae are a large family of Gram negative aerobic or facultative anaerobic oxidase negative bacteria that naturally occupy the intestinal tract of human and animals. Under certain instances, some of this enteric normal flora incidentally causes diseases such as surgical site infections (SSI) while few others are regularly pathogenic to human.

In recent years, there have been the emergence and spread of genes coding for multi-drug resistance such as extended spectrum $\beta$-lactamases (ESBL), AmpC $\beta$-lactamases, K1 $\beta$-lactamases, Klebsiella pneumoniae carbapenemases (KPC) and metallo- $\beta$-lactamases among Enterobacteriaceae and other Gram negative organisms. These $\beta$-lactamases confer resistance to most penicillins, cephalosporins and carbapenems. Many of these genes are plasmid mediated which has ensured their transfer and wide spread to many genera of bacteria in many parts of the world resulting in several outbreaks[1,2,3 ].

ESBLs are one of the most common mechanisms of antimicrobial resistance which has spread threateningly to many parts of the world[4,5,6]. These mutant plasmid mediated $\beta$-lactamases confer resistance to all first and second generation cephalosporins as well as the extended spectrum (third generation) cephalosporins. The plasmids can be transferred to other strains and species of bacteria as a result of which the coded antibiotic resistance continue to spread [7]. With Escherichia coli being the most abundant specie of Enterobacteriaceae in the gut, the tendency is that a single strain bearing antibiotic resistance plasmids soon transmits these to most members of the family in the habitat. Widespread use of third generation cephalosporins is believed to be the major driving force for the mutations that culminate in the emergence of ESBL production 
from the original TEM1, TEM2 and SHV1 cephalosporin susceptible $\beta$-lactamases in the Enterobacteriaceae family[8]. ESBL-producing organisms have also been identified with multidrug resistance to other classes of antibiotics such as aminoglycosides, ampicillins [9,10] cotrimoxazole, tetracyclines and fluoroquinolones [11] thus, further narrowing therapeutic options to few expensive antibiotics that are neither readily available nor affordable to most patients in resource poor countries.

In surgical patients, these organisms can cause serious complications such as surgical sites infections, urinary tract infection, catheter associated urinary tract infections, venous catheter infections and others with consequent septicaemia. Routine antibiotic susceptibility testing to this emerging resistance is yet to be popular in low resource settings of the world probably due to extra operational cost, time, as well as dearth of skilled manpower. The out-come thus are therapeutic failure, prolonged hospital stay, economic losses and sometimes death[12]. The continuous spread of these resistant mutants if unchecked, regrettably, portends a gradual return to the pre-antibiotic era.

In Nigeria, except for a few studies in the West[1,13] and East[14] data on the spread of these organisms is still sparse. There is therefore a paucity of data on these organisms in several parts of the country. Continuous tracking is necessary to guide rational antibiotic prophylaxis and post-operative antimicrobial intervention.

This study is aimed at defining the intestinal colonization rate of Enterobacteriaceae bearing ESBL resistance genes in surgical patients in University of Calabar Teaching Hospital using Esherichia coli as surrogate. This is necessary as part of the hospital infection control strategies toward developing a good antibiotic stewardship program.

\section{Subjects and Methods:}

2.1 Study population: The study was carried out over a five month period in the University of Calabar Teaching Hospital, Calabar Cross River State, Nigeria from June 2012 to November 2012. Ethical clearance was obtained from the University of Calabar Teaching Hospital Ethics Review Committee. Informed written consent was obtained from the patients. For Minors (patients <18years) consent was obtained from their parent or guardian. All patients booked for surgery in the hospital for which a clean or clean-contaminated wound was expected were enrolled. Patients booked with contaminated and dirty surgical wounds were excluded.

2.2 Sample collection and processing: A preformed interviewer administered questionnaire was used to collect necessary data about patient's demographics, clinical diagnosis, surgical procedure, prophylactic antibiotics, post-operative antibiotics and co-morbidity. Rectal swabs were collected from consenting patients in the ward, before surgery or within 48 hours post-surgery for surgical emergencies. Swabs were collected by inserting cotton wool swab sticks about $2.5 \mathrm{~cm}$ beyond the anal sphincter and rotated gently through the crypts. The swabs were inoculated on McConkey agar (Lab-M Ltd, Lancashire, UK) and incubated for 18 hours at $37^{\circ} \mathrm{C}$ aerobically. Escherichia coli were identified using morphologic characteristics, lactose fermentation, motility and indole production. The Antimicrobial susceptibility patterns of the isolates were determined using the Modified Kirby-Bauer disc diffusion method according to the Clinical and Laboratory Standard Institute (CSLI) guidelines [15]. Mueller Hinton agar (Lab-M Ltd Lancashire, UK) and Oxoid antibiotics discs (Oxoid Ltd, Cambridge, UK) were used. E. coli isolates with $30 \mu \mathrm{g}$ ceftriaxone disc zone of inhibition $\leq 27 \mathrm{~mm}$ and $30 \mu \mathrm{g}$ ceftazidime disc zone $\leq 22 \mathrm{~mm}$ were suspected to be ESBL producing and subjected to Double Disc Synergy ESBL confirmatory test [16] as demonstrated in Figure 1. Morphologic classification of ESBL to either the CTX-M or TEM/SHV types was made if it was detected by either cefotaxime or ceftazidime respectively [16].

2.3 Quality controls: American Type Culture Collection (ATCC) 25922 Escherichia coli was used as susceptibility test control and ESBL negative control. Locally isolated ESBL producing E. coli designated AFU11 was used as ESBL positive control.

2.4 Data analysis: All data were analyzed using Statistical Package for Social Sciences (SPSS) version 19.0 (SPSS Inc. Chicago, USA). All data were categorical and presented as \% (n/sample size). Pearson's correlations and Chi square test were used to evaluate relationships. All $P$-values were 2 -sided and a $P$-value $\leq 0.05$ was considered to be statistically significant.

\section{Results:}

A Total of 192 patients were enrolled into the study. The patients were drawn from Adult surgery 47.9\% (92/192), Paediatrics surgery 36.5\% (70/192), Obstetrics and gynaecology 15.6\% (30/192). Elective surgical cases were $89.6 \%$ (172/192) while $10.4 \%$ (20/192) had emergency procedures.

Surgical procedures performed on the patients included: Abdomino-pelvic resection of colorectal Cancer $(n=1)$, Uterine Adhesiolysis( $(n=4)$, Appendectomy $(n=15)$, Orchidopexy $(n=2)$, Excision biopsy $(n=25)$, 
Excision of cystic hygroma $(n=2)$, Examination under anaesthesia $(n=2)$, Hydrocoelectomy $(n=6)$, Haemorhoidectomy $(n=3)$, Herniorhaphy/herniotomy $(n=73)$, Laparotomy $(n=22)$, nephrolitotomy $(n=2)$, Open reduction and internal Fixation $(n=2)$, Prostatectomy $(n=3)$, Parathyroidectomy $(n=1)$, Posterior urethral valve excision( $n=2)$, Urethroplasty $(n=2)$, Hystero-Salpingography $(n=2)$, Caesarian $\operatorname{section}(n=18)$, Total Abdominal hysterectomy $(\mathrm{n}=6)$.

The prophylactic antibiotic prescription pattern is as shown in Table 1. A Total of $67.7 \%$ (130/192) of the patients received a prophylactic antibiotic which was continued for a minimum of five days after surgery. All the patients who did not receive any antibiotic were in the paediatric subgroup (88.6\% (62/70)). Ceftriaxone was the most favoured antibiotic. It was used in combination with metronidazole in $63.1 \%(82 / 130)$ and alone in $7.7 \%(10 / 130)$ of the cases.

Antimicrobial susceptibility of the rectal E. coli isolates revealed that $39.6 \%(76 / 192)$ were resistant and $3.1 \%(6 / 192)$ had intermediate susceptibility to ceftriaxone. Ceftazidime resistance was observed in $40.6 \%$ $(78 / 192)$ while $4.2 \%(8 / 192)$ showed intermediate susceptibility. ESBL production was demonstrated in $27.1 \%$ $(52 / 192)$ of the isolates. Phenotypic evaluation of genetic mechanisms showed that $61.5 \%(32 / 52)$ were detected by both cefotaxime and ceftazidime which suggest CTX-M, SHV, or TEM-type ESBL, 30.8\% (16/52) were detected with cefotaxime alone suggesting CTX-M-type whereas 7.6\% (4/52) were detected with ceftazidime alone suggestive of the TEM or SHV-type ESBL (Table 2).

Comparison of susceptibility of ESBL positive and negative isolates to other classes of antimicrobials (Table 3) showed that all isolates were susceptible to Meropenem. ESBL positive isolates were more likely to be resistant to Gentamicin $(\chi 2=15.190 ; \mathrm{df}=2 ; P=0.001)$, Ciprofloxacin $\quad(\chi 2=35.829 ; \mathrm{df}=2 ; P<0.0001)$ and Cotrimoxazole $(\chi 2=9.229 ; \mathrm{df}=1 ; P=0.001)$. A higher level of resistance to Amoxicillin-clavulanate $57.8 \%$ was observed amongst the ESBL-positive organisms however, there was no statistically significant difference between the ESBL positive and negative $(47.1 \%)$ group $(\chi 2=3.743 ; \mathrm{df}=2 ; P=0.154)$ (Table 3$)$

Younger patients were more likely to be colonized as they was a negative correlation between age and ESBL colonization which was statistically significant (Pearson's correlation $=-0.196 ; P=0.006)$. None of the patients older than 60 years was colonized while patients less than 9 years were more preponderant (Figure.2).

Evaluation of prophylactic antibiotic coverage of the patients bearing rectal ESBL showed that $50.0 \%$ $(26 / 52)$ received ceftriaxone with or without metronidazole, $38.5 \%(20 / 52)$ which were also all of the colonized, paediatrics patients had no antibiotics while cefuroxime alone, cefuroxime with metronidazole and ofloxacin alone were given to $3.8 \%(2 / 52)$ of patients each.

\section{Discussion:}

This study evaluated the rate of ESBL colonization of surgical patients in the University of Calabar Teaching Hospital using Escherichia coli as surrogate organism. E. coli was used because it is the most predominant Enterobacteriaceae in the gut and therefore the most likely to be transferred from the gut to other sites or to other patients. The $27.1 \%$ rectal ESBL carriage rate recorded in this work is similar to $22.1 \%$ recorded amongst paediatric patients on first day of hospitalization in Madagascar [17] and 26.8\% in Buenos Aires, Argentina [18] but relatively lower than 32\% among clinical isolates reported in India [5]. Since this resistance mechanism is plasmid mediated, there is a tendency for its spread in the community and more especially in hospital settings. This will further undermine the usefulness of third generation cephalosporins, several penicillins as well as other classes of antibiotics such as aminoglycosides, quinolones, sulfamethoxazole/trimethoprim due to co-resistance as was observed here and have also been reported elsewhere $[9,10,11]$. The other resistance mechanisms which may have accounted for the remaining $50 \%$ of non ESBL resistance to third generation cephalosporins may have been plasmid or chromosomal ampC and $\mathrm{K} 1 \beta$ lactamases. Apart from chromosomal ampC, the others are also plasmid mediated and thus could be transferred from one Enterobacteriaceae to another thereby compounding the problem of antibiotic resistance. Most chromosomal ampC bearing Enterobacteriaceae may also carry ESBL resistance mechanisms which will require the demonstration of clavulanic effect with cefepime and resistance to cefoxitin [16] which were not available to us at time of study. The failure of routine susceptibility testing techniques to include testing for ESBL and other resistance mechanisms in Enterobacteriaceae may lead to poor surgical outcome with increased morbidity and possibly mortality.

The relative predominance of the CTX-M subtypes of ESBL(30.8\%) obtained in this study is similar to values of 29.3-50.6\% recorded in Thailand [19] but significantly different from value of $11.3 \%$ obtained in Birmingham, UK [ 20]. CTX-M ESBL genotype is tending towards a global pandemic, with intercontinental travel as a major enhancing factor [21]. Nigeria enjoys rich medical tourism with India, one of the countries with high ESBL carriage rate with a predominance of CTX-M genotypes [5].

There was a higher propensity for an ESBL producing isolate to be resistant to other classes of antimicrobials such as the quinolones $(57.7 \%)$ and aminoglycoside $(26.9 \%)$. These findings are similar to values of $40 \%$ for a quinolone and $14 \%$ for an aminoglycoside from a study in Morocco [22]. Empirical treatment 
options for these organisms are therefore limited. Treatment of ESBL producing organisms with antibiotics to which they are resistant will lead to treatment failure, waste of finances and high mortality.

It was observed in this study that most adult patients received prophylactic antibiotics administered before surgery and continued empirically till about the $5^{\text {th }}$ post-operative day. Evaluation of prescription pattern showed a higher use in adult surgeries than paediatric surgeries. There was the highest proclivity towards cephalosporins particularly ceftriaxone. Since surgical sites infections are more likely to originate from patients flora $_{2}$ the high level of resistance to cephalosporins by rectal E. coli observed in this study means their prophylactic use and inappropriate empirical use in this patients would not have been of any clinical benefit. With the plasmid mediated spread of ESBL and other $\beta$-Lactamase resistance mechanisms [10], the usefulness of this antibiotics is bound to get worse with the coming decades. The high rate of resistance to cephalosporins which are most predominantly prescribed for surgical prophylaxis further selects for mutants thus drive emergence of antibiotic resistance.

Established risk factors for ESBL colonization are previous hospital admission or visit and cephalosporin use [23]. The correlation of ESBL colonization with younger age observed in this study had also been observed elsewhere [24, 25]. Among infants and the very young with no history of previous hospital admissions and cephalosporin use observed in this study, colonization most probably originated from maternal or environmental flora [26]. Several studies have shown that the feacal colonization of newborns with ESBLproducing organisms take origin from maternal vagina during delivery with subsequent spread to care-givers and the environment $[27,28]$.

\section{Conclusion}

This study has shown that there is a circulating pool of ESBL in several patients seeking care in the hospital. ESBL colonization of children may result in horizontal spread among peers, with risk of outbreaks in paediatric wards. High impact intervention strategies recommend routine screening of surgical patients preoperatively for Methicillin Resistant Staphylococcus aureus (MRSA) [29]. With the high prevalence of ESBL observed in this study and many others, $[5,17,18]$ a pre-surgery rectal screening for ESBL as well as other resistance mechanisms such as ampC, and $\mathrm{K} 1 \beta$-lactamases, is therefore imperative. Patients with pan-resistant isolates could also be evaluated for carbapenamases such as KPC and metallo- $\beta$-lactamases. This will provide ground for high index of suspicion, guide rational prophylaxis and empirical therapy since most health-care associated infections in surgical patients usually emanate from patient own flora [30]. In Nigeria, although there are regulations on antibiotics procurement and administration, there are rarely enforced. All brands of antibiotics can be purchased over the counter and in market places. Community abuse of antibiotics and irrational hospital use as evident in this study accounts for the high carriage rate of ESBL and antibiotic resistance recorded in this study. This is also buttressed by the $100 \%$ susceptibility still observed for Meropenem in this setting. Carbapenems are new entrants into the market. They are still relatively expensive and only injectable forms are available which makes community abuse less likely. However, irrational use in healthcare facilities and import of metallo $\beta$-lactamases through medical tourism may soon drive resistance. Continuous monitoring, formulary restriction, functional antibiotic stewardship programmes and strict regulations as has been demonstrated elsewhere [31] will therefore be necessary to curtail this gradual slip into the pre-antibiotic era.

Conflict of interest:

We declare that we have no conflict of interest.

\section{Acknowledgement:}

This research was coordinated by Save Lives Initiative (savelivesinitiative@gmail.com), a not for profit organization which supports research efforts of young scientists.

\section{References:}

[1] Egbebi, AO, Famurewa OJ. Prevalence of extended spectrum beta-lactamase (ESBL) production among Klebsiella isolates in some parts of South West Nigeria. Microbiol Biotech Res 2011;1(2):64-68.

[2] Lopez- cerero L, de Cueto M, Saenz C, Navarro D, Velasco D, et al. Meningitis caused by CTX-M-32 -producing E.coli isolate. J Med Microbiol 2008;57:1303-1305.

[3] Iregbu KC, Anwaal U. Extended spectrum $\beta$-lactamase producing Klebsiella pneumoniae septisemia outbreak in the neonatal intensive care unit of a tertiary hospital in Nigeria. Afr J Med Sci 2007;36 (3):225-228.

[4] Metri BC, Jyothpi P, Peerapur BV. ESBLs among Enterobacteraceae. J Clin Diagn Res 2011;5(3):470-475.

[5] Shobha KL, Gowrish RS, Sugandhi R, Sreeja CK. Prevalence of Extended Spectrum Beta-Lactamases in Urinary Isolates of Escherichia coli, Klebsiella and Citrobacter Species and their Antimicrobial Susceptibility Pattern in a Tertiary Care Hospital. Indian J Pract Doc 2005;3(6):50-51.

[6] Kiratisin P, Apisarnthanarak A, Laesripa C, Saifon P. Molecular characterization and epidemiology of extended-spectrum $\beta$ lactamase producing Escherichia coli and Klebsiella pneumoniae isolates causing health care-associated infection in Thailand, where the CTX-M family is endemic. Antimicrob Agents Chemother 2008;52(8):2818-2824.

[7] Olowe AO, Aboderin BW. Detection of extended spectrum $\beta$-lactamase producing strain of (Escherichia coli) and (Klebsiella sp.) in a tertiary health center in Ogun state. Int J Trop Med 2010;5(3):62-64 
[8] Chaudhary U, Aggarwal R. Extended spectrum $\beta$-lactamases (ESBL) - An emerging threat to clinical therapeutics. Indian J Med Microbiol 2004;22(2):75-80.

[9] Viswanathan R, Sigh KA, Basu S, Chatterjee S, Sardar S, et al. Multi-drug resistant Gram negative bacilli causing early neonatal sepsis in India. Arch Dis Child Fetal Neonatal Ed 2012;97(3):182-187

[10] Shin SY, Kwon KC, Park J W, Song JH, Ko YH, et al. Characteristics of aac(6')-Ib-cr Gene in Extended-Spectrum $\beta$-LactamaseProducing Escherichia coli and Klebsiella pneumoniae Isolated from Chungnam Area. Korean J Lab Med 2009;29:54 1-50.

[11] Morosini MI, García-Castillo M, Coque TM et al. Antibiotic coresistance in extended-spectrum- $\beta$-lactamase-producing Enterobacteriaceae and in vitro activity of tigecycline. Antimicrob Agents Chaemother 2006;50(8):2695-2699.

[12] L'apsley HM, Vogels R Quality and cost impacts: prevention of post-operative clean wound infections. Int J Health Care Qual Assur 1998;11: 222-231.

[13] Okesola OA, Adeniji TW Pattern of Beta lactamase production among clinical isolates of Proteus species in Western Nigeria. World J Med Sci 2010;5(4):94-97.

[14] Iroha IR, Adikwu MU, Esimone CO, Aibinu I, Amadi ES Extended spectrum Beta lactamases (ESBL) in E. coli isolated from a tertiary hospital in Enugu state Nigeria. Pak J Med Sci 2009;25:279-288.

[15] Clinical and Laboratory Standard Institute. Performance Standard for Antimicrobial Susceptibility Testing; Twenty-Second Informational Supplement. CLSI document M100-S22 2012. Wayne, PA.

[16] Livermore DM, Brown DF. Detection of $\beta$-lactamases mediated resistance. J Antimicrob chemother. 2001;48(s1):59-64.

[17] Andriatahina T, Randrianirina F, Hariniana ER, Talarmin A, Raobijaona H et al. High prevalence of fecal carriage of extendedspectrum $\beta$-lactamase-producing Escherichia coli and Klebsiella pneumoniae in a pediatric unit in Madagascar. BMC Infect Dis 2010;10:204. http://www.biomedcentral.com. Accessed 2/4/2013.

[18] Villar HE, Baserni MN, Jugo MB Feacal carriage of ESBL-producing Enterobacteriaceae and Carbapenem resistant Gram-negative bacilli in community settings. J Infect Dev Ctries 2013;7(8):630-634.

[19] Luvsansharav UO, Hirai I, Niki M, Sasaki T, Makimoto K et al. Analysis of risk factors for a high prevalence of extended-spectrum b-lactamase-producing Enterobacteriaceae in asymptomatic individuals in rural Thailand. J Med Microbiol 2011;60: 619-624.

[20] Wickramasinghe NH, Xu L, Eustace A, Shabir S, Saluja T, et al. High community faecal carriage rates of CTX-M ESBL-producing Escherichia coli in a specific population group in Birmingham, UK. J Antimicrob Chemother 2012;67(5):1108-1113.

[21] Woerther PL, Burdet C, Chachaty E, Andremont A. Trends in Human Fecal Carriage of Extended-Spectrum <beta>-Lactamases in the Community: Toward the Globalization of CTX-M. Clin Microbiol Rev 2013;26(4):744-758.

[22] Malki Fatima EL, Bouraissi Meriem EL, Said B. Plasmid Mediated Quinolones Resistance ESBL-Enterobactériaceae in Moroccan. Pharmaceut Anal Acta 2012;S15. http://www.omicsonline.org. Accessed 2/4/2013.

[23] Pasricha J, Koessle T, Harbarth S, Schrenzel J, Camus V et al. Carriage of extended-spectrum beta-lactamase-producing enterobacteriacae among internal medicine patients in Switzerland. Antimicrob Res Infect Control 2013;2:20. http://www.aricjournal.com./content/2/1/20. Accessed 17/7/ 2013.

[24] Schaumburg E, Alabi A, Kokou C, Grobusch MP, Kaba H et al. High burden of extended spectrum $\beta$-lactamase-producing Enterobacteriaceae in Gabon. J Antimicrob Chemother 2013;68(9): 1240-1243.

[25] Dunais B, Blanc V, Bruno P, Nicholas-Chanoine M, Leflon-Guibout V, et al. Extended spectrum $\beta$-lactamase producing Enterobacteriaceae in children's daycare centres: a cross-sectional study of faecal carriage in Southeastern France. Conference proceedings of the European society of clinical microbiology and infectious diseases, Berlin, Germany, 27-30 ${ }^{\text {th }}$ April 2013. Accessed 29/9/2013.

[26] Gaze WH, Abdouslam N, Hawkey PM, Wellington EM. Incidence of class 1 integrons in a quaternary ammonium compoundpolluted environment. Antimicrob Agents Chemother 2005; 49(5):1802-1807.

[27] Tschudin-Sutter S, Frei R, Battegay M,Hoesli I, Widmer AF. Extended spectrum $\beta$-lactamase producing E. coli in a neonatal care unit. Emerg Infect Dis 2010; 169(11):1758- 1760.

[28] Dubois V, De Barbeyrac B, Rouges A, Arpin C, Coulange L et al. (2010) CTX-M-producing Escherichia coli in a maternity ward: a likely community importation and evidence of mother-to-neonate transmission. J Antimicrob Chemother 65(7):1368-1371.

[29] Harbarth S, Fankhauser C, Schrenzel J, Christenson J, Gervaz P. et al. Original Contribution .Universal Screening for MethicillinResistant Staphylococcus aureus at Hospital Admission and Nosocomial Infection in Surgical Patients. JAMA 2008;299(10):11491157.

[30] Nicholas RL. Surgical wound infection. Am J Med 1991;91:54-63.

[31] Cheng AC, Turnidge J, Collignon P, Looke D, Barton M, Gottlieb T Control of Fluoroquinolone Resistance through Successful regulation, Australia. Emerg Infect Dis 2012;18(9):1453-1460.

Table1: Prophylactic and Post surgery antibiotic prescription pattern in UCTH

\begin{tabular}{|c|c|c|c|c|c|c|c|c|}
\hline & & & & $\begin{array}{l}\text { Antibiotic } \\
\text { number(\%) }\end{array}$ & & & & \\
\hline & $\overline{\mathrm{CRO}}$ & $\mathrm{CRO}+\mathrm{MET}$ & $\mathrm{CFU}$ & CFU+MET & CIP & $\mathrm{CIP}+\mathrm{MET}$ & OFX & $\overline{\text { NONE }}$ \\
\hline $\begin{array}{l}\text { Unit } \\
\text { Adult surgery } \\
\mathrm{n}=92\end{array}$ & $0(0.0)$ & $62(67.4)$ & $0(0.0)$ & $2(2.2)$ & $2(2.2)$ & $24(26.1)$ & $2(2.2)$ & $0(0.0)$ \\
\hline $\begin{array}{l}\text { Paed surgery } \\
n=70\end{array}$ & $0(0.0)$ & $8(11.4)$ & $0(0.0)$ & $0(0.0)$ & $0(0.0)$ & $0(0.0)$ & $0(0.0)$ & $62(88.6)$ \\
\hline $\begin{array}{l}\text { Obst/gyn } \\
n=30\end{array}$ & $10(33.3)$ & $12(40.0)$ & $4(13.3)$ & $2(6.7)$ & $0(0.0)$ & $2(6.7)$ & $0(0.0)$ & $0(0.0)$ \\
\hline Total $n=192$ & $10(5.2)$ & $82(42.7)$ & $4(2.1)$ & $4(2.1)$ & $2(1.0)$ & $26(13.5)$ & $2(1.0)$ & $62(32.3)$ \\
\hline
\end{tabular}

CRO-ceftriazone; CIP-Ciprofloxacin; CFU-Cefuroxime; MET-Metronidazole; OFX-Ofloxacin; Paed-

Paediatrics; Obst/gyn-Obstetrics/gynecology 

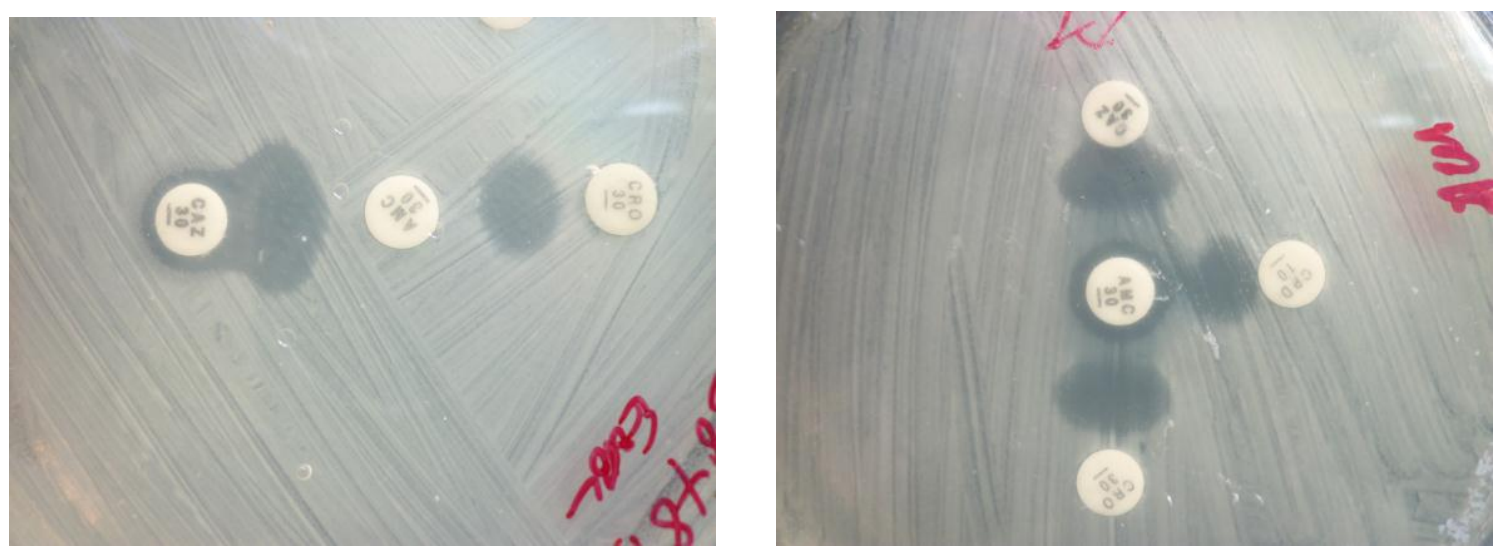

Figure 1: Demonstration of Clavulanic effect in the Double Disc Synergy ESBL Confirmatory test.

Table 2: Distribution of phenotypically adjudged genotypic mechanisms of ESBL mediated resistance to cephalosporins in UCTH Calabar.

\begin{tabular}{lcccc} 
& & \multicolumn{3}{c}{ ESBL type } \\
& $\begin{array}{c}\text { SHV/CTX-M/TEM } \\
\mathrm{n}=52\end{array}$ & $\begin{array}{r}\text { CTX-M } \\
\mathrm{n}=52\end{array}$ & $\begin{array}{c}\text { SHV/TEM } \\
\mathrm{n}=52\end{array}$ & $\begin{array}{c}\text { Total } \\
\mathrm{n}=192\end{array}$ \\
\hline Number(\%) & $32(61.5)$ & $16(30.8) 4(7.6)$ & $52(27.1)$ & \\
\hline
\end{tabular}

Table 3: Pattern of antimicrobial susceptibility of ESBL positive and negative intestinal $E$. coli isolates to other classes of antimicrobials in UCTH Calabar.

\begin{tabular}{ccc}
\hline ESBL Positive & ESBL Negative & Total \\
no(\%) & no(\%) & no(\%) \\
$\mathrm{n}=52$ & $\mathrm{n}=140$ & $\mathrm{n}=192$
\end{tabular}

Antimicrobial

\section{Ciprofloxacin $(5 \mu \mathrm{g})$}

Resistant

Intermediate

Susceptible

$\chi 2=35.829 ; \mathrm{df}=2 ; P<0.0001$

Gentamicin $(10 \mu \mathrm{g})$

Resistant

Intermediate

Susceptible

$\chi 2=15.190 ; \mathrm{df}=2 ; P=0.001$

Cotrimoxazole $(1.25+23.75 \mu \mathrm{g})$

Resistant

Intermediate

Susceptible

$\chi 2=9.229 ; \mathrm{df}=1 ; P=0.001$

$14(26.9)$
$4(7.7)$
$34(65.4)$

$$
\text { 10(7.1) }
$$

$130(67.7)$

Meropenem $(10 \mu \mathrm{g})$

$\begin{array}{lccc}\text { Resistant } & 0(0.0) & 0(0.0) & 0(0.0) \\ \text { Intermediate } & 0(0.0) & 0(0.0) & 0(0.0) \\ \text { Susceptible } & 52(100.0) & 140(100.0) & 192(100.0)\end{array}$


Amoxicillin/Clavulanate (20-10 $\mathrm{g}$ )

$\begin{array}{lclr}\text { Resistant } & 28(53.8) & 66(47.1) & 94(49.0) \\ \text { Intermediate } & 8(15.4) & 12(8.6) & 20(10.4) \\ \text { Susceptible } & 16(30.8) & 62(44.3) & 78(40.6)\end{array}$

$\chi 2=3.743 ; \mathrm{df}=2 ; P=0.154$

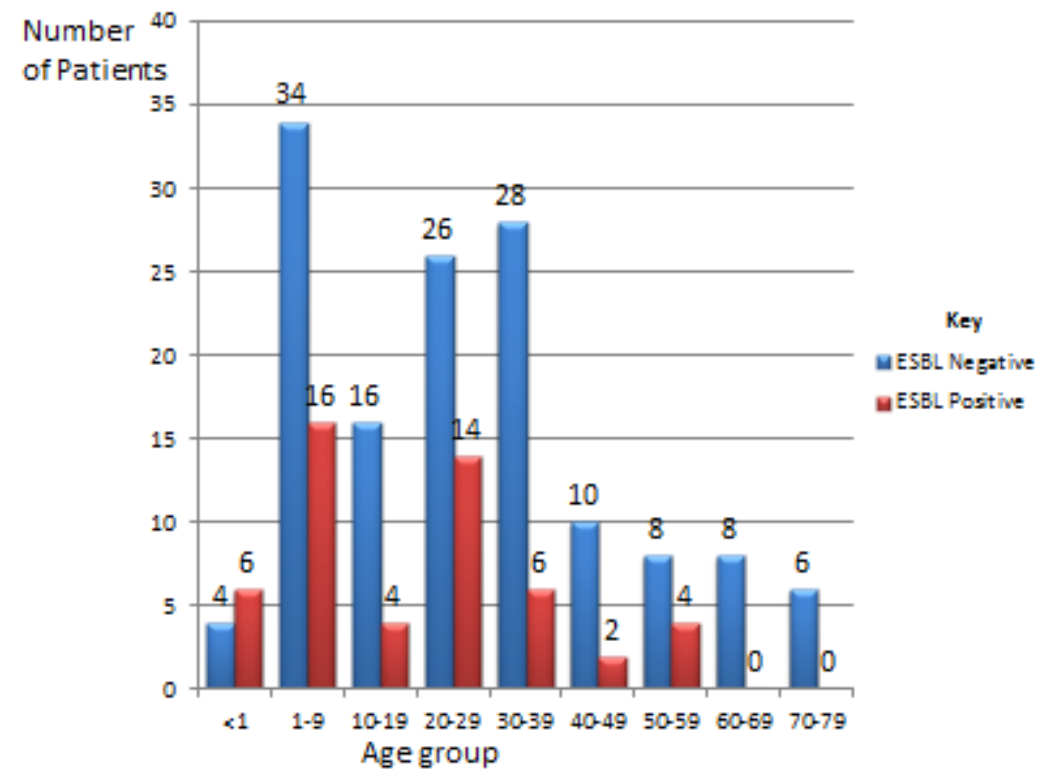

Pearson's correlation $=-0.196 ; P=0.006$

Figure 2. Age distribution of surgical patients with and without rectal ESBL- producing Escherichia coli in UCTH, Calabar. 